$10 \mid 2021$

Intelligence artificielle, pratiques sociales et politiques publiques

\title{
Promotion du civisme fiscal en Afrique grâce au téléphone mobile
}

Cas d'utilisation du téléphone portable au Burundi

Promotion of tax compliance in Africa through the mobile phoneMobile phone use, Burundi case.

Fiacre Muhimpundu

\section{OpenEdition}

\section{Journals}

Édition électronique

URL : https://journals.openedition.org/ctd/5088

DOI : $10.4000 /$ ctd. 5088

ISSN : 2491-1437

Éditeur

Chaire Unesco Pratiques émergentes en technologies et communication pour le développement

Édition imprimée

ISBN : 2491-1437

\section{Référence électronique}

Fiacre Muhimpundu, "Promotion du civisme fiscal en Afrique grâce au téléphone mobile », Communication, technologies et développement [En ligne], 10 | 2021, mis en ligne le 18 mai 2021, consulté le 27 mai 2021. URL : http://journals.openedition.org/ctd/5088 ; DOI : https://doi.org/ $10.4000 /$ ctd. 5088

Ce document a été généré automatiquement le 27 mai 2021.

Communication, technologies et développement 


\title{
Promotion du civisme fiscal en
} Afrique grâce au téléphone mobile

\author{
Cas d'utilisation du téléphone portable au Burundi \\ Promotion of tax compliance in Africa through the mobile phoneMobile phone \\ use, Burundi case.
}

Fiacre Muhimpundu

\section{NOTE DE L'ÉDITEUR}

Uguteza imbere umugenzo mwiza wo gutanga amakori ata gahato hakoreshejwe imihora ya telephone ngendanwa

Ico kibazo cifashe gute mu Burundi?

Ubuhinga ngurukanabumenyi bwarazanye impinduka igaragara mu nyifato

y'abatangakori mu bihugu bifise ubutunzi bubayabaye, harimwo Afrika iri musi ya Sahara. Naho ibiharuro vyerekana ko Afrika ikiri kure mu bice vy'isi bimaze guteza imbere no gukamisha ubuhinga ngurukanabumenyi, itelefone ngendanwa yo yatanguye gukwiragizwa henshi muri Afrika mu mpera y'imyaka ya 90, bikaba vyagiye birongerekana mu myaka yakurikiye.

Ukuriha amakori n'amatagisi biciye muri telefone ngendanwa, nico kibazo iki cigwa caciye hirya no hino, aho vyabonetse ko ukuriha biciye muri ubwo buryo vyari vyazanye ukworoherezwa kudasanzwe ariko bikagarukira ku mugwi muto w'abatangakori (baba mu bisagara), yamara abandi benshi bakidandaza bike akaba ari nabo benshi bakwiragiye ku mitumba n'udusoko dutoduto mu ntara. Abo badandaza rero bibereyeho ikori batariha bikitse inyuma y'ubuhinga buke ikigo gitoza amaori gifise ngo gishobore kumenya aho bari, kibandike congere kibatoze ikori. Aho rero niho hagaragarira uruhara rw'ubuhinga ngurukanabumenyi.

Igica kiba, nuko ku ruhande rumwe, ukumeyesha no kwakira amakuru afatiye mu guhimiriza abantu gutanga amakori neza ata gahato bitaba bigikwiye hatagiyemwo ubuhinga ngurukanabuleyi buza gusahiriza. Ku rundi ruhande, ubwo buhinga buba 
impfagusa iyo butakiriwe n'abo bwagenewe gufasha ngo babukoreshe babunezererewe. Ivyo navyo bikunda aruko habanje kuba ihimiriza.

Iki cigwa kigerageza kwerekana impinduka nziza zozanwa na telefone ngendanwa nk'igikoresho co guteza imbere umugenzo mwiza wo gutanga amakori ata gahato biciye ku kamwemwe abatangakori bokwumva babonye borohewe kandi binyaruka iyo bariko barahanahana amakuru n'ikigo gitoza amakori, bakayatanga bitabavunye. itelefone, ugukora ibisabwa ; ukworoherezwa

\section{Introduction}

1 On ne compte plus les articles de presse et les articles à caractère scientifique sur la téléphonie mobile et son impact sur le développement de l'Afrique.

2 En Afrique subsaharienne, le taux de pénétration de la téléphonie mobile varie énormément d'un pays à l'autre. Au Niger et en République centrafricaine, il n'atteint pas $25 \%$ et en Érythrée il est estimé à $9 \%$. À l'île Maurice, aux Seychelles, au Botswana et en Afrique du Sud, les taux sont proches des $70 \%{ }^{3} \mathrm{Au}$ Burundi, le téléphone mobile, avec son coût faible et son ubiquité, est l'outil désormais accessible à toute heure et en tout lieu pour le paysan des collines. Le téléphone mobile s'érige en outil indispensable en faveur de groupes isolés et de zones reculées. Il ne demande en outre pas un niveau d'alphabétisation et de maîtrise technique élevé.

3 Selon Annie Chéneau-Loquay ${ }^{4}$, la réussite de la téléphonie mobile en Afrique peut être expliquée par la mise en œuvre du modèle néolibéral à l'échelle mondiale, mais aussi par les capacités d'adaptation des opérateurs internationaux à la demande locale ; ils ont su changer de modèle économique pour répondre aux besoins d'une population pauvre qui cherche à minimiser ses dépenses.

4 Les échanges (notamment financiers) à travers le téléphone mobile deviennent une routine pour un paysan lamda sur les collines et une aubaine de petites affaires pour les micro contribuables. Ceux-ci ouvrent en un laps de temps des comptes de transfert d'argent par téléphone mobile.

5 En Afrique subsaharienne, on comptait fin 2018, 122 millions de comptes actifs (dernier mois) pour 400 millions de comptes enregistrés, plus que partout ailleurs dans le monde (GSMA, 2018). ${ }^{5}$

6 Ces dernières années, l'utilisation des téléphones portables, des ordinateurs et d'Internet a connu un développement sans précédent en Afrique ${ }^{6}$. L'adoption rapide de ces technologies par les Africains et leur prolifération sur le continent ont suscité une vaste littérature.

7 L'étude menée par le $\mathrm{FMI}^{7}$ et publiée en avril 2020 portant sur la transformation numérique en Afrique subsaharienne évoque une valeur ajoutée dans la collecte des recettes réalisée grâce à la dématérialisation des procédures de déclaration et de paiement des impôts et taxes. Les chiffres montrent que les pays dont le niveau de pénétration d'Internet et le nombre d'abonnements mobiles sont plus élevés enregistrent aussi davantage de recettes. Une augmentation de la transformation numérique en Afrique subsaharienne entraîne une augmentation des recettes intérieures pouvant aller jusqu'à 2,1 points de pourcentage du PIB. Les pays adoptant la télédéclaration ont également tendance à être plus efficients en matière de collecte de la taxe sur la valeur ajoutée, tandis que près de la moitié des pays de la région ont 
enregistré une amélioration considérable de l'efficacité de la perception grâce à la dématérialisation, ce qui engendre la simplification de l'administration fiscale. L'abandon du paiement des impôts par chèque et en espèces peut réduire les coûts de transport, de main d'œuvre et de transaction. En 2014, par exemple, l'Autorité des recettes publiques du Kenya (Kenya Revenue Authority, KRA) a introduit l'iTax, une administration fiscale intérieure automatique et totalement intégrée qui permet aux contribuables de payer à partir de leurs appareils mobiles. La KRA a ainsi pu mettre en place un suivi et un audit en temps réel des recettes, ce qui lui a permis de réduire progressivement le coût du recouvrement de l'impôt (Ndung'u, 2017). Depuis 2016, la transformation numérique des services fiscaux en Afrique du Sud, qui a automatisé le traitement et la gestion des risques, a considérablement réduit les pertes de temps et la charge administrative associées au calcul de l'impôt et à l'évaluation en douane. Aujourd'hui, la plupart des calculs de l'impôt sur le revenu (95\%) sont effectués en 3 secondes, contre 180 jours en 2006 (FMI, 2018c).

8 La communication pour la promotion du civisme fiscal ne peut être efficace sans la facilitation des affaires. Cette facilitation est rendue possible par les Technologies de l'Information et de Communication.

9 Au Burundi, l'office burundais des recettes (le nom de l'actuelle Administration fiscale et douanière du Burundi) a développé des programmes de facilitation des contribuables dans le domaine des douanes, mais la conformité fiscale entraîne toujours des coûts considérables en raison du manque d'installations. Ainsi, la sensibilisation menée se heurte toujours à une barrière qui consiste en la difficulté de l'administration fiscale à faciliter les contribuables lorsqu'ils sont en train d'honorer leurs obligations fiscales après avoir parcouru de longues distances pour payer les impôts. Tout de même, des efforts ont été faits pour faciliter les affaires, mais il reste encore beaucoup à faire. La décision la plus récente allant dans le sens de la décentralisation des services étant le déploiement du service du transfert de propriétés au niveau de trois régions fiscales intérieures dans les provinces de Gitega, Muyinga et Ngozi.

Une attitude inattendue cependant: malgré la mise en place de moyens informatiques notamment le système électronique de suivi du fret utilisant des balises attachées aux camions-cargo, les données de l'administration fiscale burundaise montrent un faible intérêt des contribuables pour ces facilités. Le suivi des dossiers dans le processus de dédouanement par message téléphonique (SMS), qui est une des facilités optionnelles offertes gratuitement aux utilisateurs étant une illustration notoire de ce manque d'adhésion ${ }^{8}$. Cette attitude pourrait être expliquée par des théories traitant de la communication pour le changement social, des théories de l'usage et des théories des valeurs.

11 La théorie des usages, par exemple, pourrait expliquer comment les contribuables perçoivent les technologies de l'information et les dispositifs mis à leur disposition pour une meilleure conformité fiscale. En effet, l'arrivée des nouvelles technologies de l'information et de la communication (NTIC) a "remis au goût du jour la question du rapport entre culture et technologie » (Mercier, 1993), les interactions entre société et innovations, l'introduction des innovations dans la société, leur utilisation et acculturation “.

12 L'appropriation du comportement souhaité ou son rejet peut avoir pour origine la perception de l'outil intermédiaire entre le contribuable et l'État dans l'accomplissement de ses obligations fiscales, sans parler de la menace qu'il peut 
ressentir provenant du nouveau dispositif qui lui est imposé. Le choix de cette étude est imposé par le contexte de recherche où des innovations ont été introduites dans l'administration fiscale grâce à des partenariats avec les banques et l'opérateur téléphonique local dénommé LUMITEL ${ }^{9}$.

13 L'objectif de cette étude est de découvrir la relation entre les contribuables et le téléphone mobile comme une technologie d'information et de communication mise à leur disposition pour payer les impôts et taxes dans les pays à pôles semi-urbains comme le Burundi.

Basé sur une revue de la littérature, des statistiques et des conversations, cet article examine le degré d'adoption du téléphone mobile par les contribuables burundais comme moyen de leur facilitation et par conséquent de promotion du civisme fiscal. Il souligne les limites des changements observés et conclut que les contribuables burundais n'ont pas adopté la facilitation par le téléphone mobile, qui est plutôt perçu comme un outil qui accélère plus la collecte des recettes que la facilitation des contribuables.

15 La présente étude cherche à trouver réponse à la question suivante : pourquoi les contribuables burundais continuent-ils à faire la queue lors des périodes de paiement des impôts et taxes alors qu'ils pourraient le faire au moyen d'un téléphone mobile?

Partant des arguments ci-haut développées, l'étude se donne une hypothèse à vérifier qui est libellée comme suit: les contribuables burundais ne sont pas pour autant facilités avec l'introduction du téléphone mobile comme moyen de paiement des impôts et taxes.

\section{Positionnement théorique}

17 Ce travail s'inscrit dans le domaine de la communication pour le changement social d'une part et de la sociopolitique des usages d'autre part.

\section{Communication pour le changement social}

Ce courant théorique développé par divers auteurs insiste sur l'importance du contenu et des capacités de persuasion des messages dans le comportement social (Schramm). L'idée défendue ici est qu'il ne suffit pas de combler le «fossé numérique ». Un simple accès à l'information et aux médias ne conduirait pas nécessairement à des résultats satisfaisants et une attention particulière devrait être portée à la qualité de l'information, ainsi qu'au contenu des messages (Schramm, 1964a, 1964b [2006]; Kiyindou, 2006).

Le communicateur ne pourra jamais décider pour ceux qui ont besoin de développement, - il ne peut qu'aider à organiser un débat et une prise de décision en faveur d'un nouveau comportement $(\text { Schramm })^{10}$. La communication pour le changement social met également en exergue le rôle de la communication interpersonnelle par rapport aux médias de masse. Alors que les médias ne peuvent transmettre que des informations, les canaux de communication interpersonnels seraient beaucoup plus susceptibles de susciter l'intérêt et la motivation pour le changement et l'intérêt des "leaders d'opinion » (Rogers) ${ }^{11}$. Si la technologie n'est plus reconnue comme le seul facteur de développement, les facteurs "en amont» 
(Andreasen, 1997; Andreasen et Herzberg, 2005), e. g. les facteurs institutionnels et structurels macro-sociaux sont pris en compte (Vaswani et al., 2005 ; Standing, 2004). La communication pour le développement est également beaucoup plus « contextuelle» et " participative », à travers l'analyse des besoins et l'écoute de la « voix des pauvres» (Standing, 2004 ; Talukdar et al., 2005).

L'utilisation du marketing social présente à son tour certains avantages. Son approche est plus nuancée tant par rapport aux publics (segmentation des utilisateurs) que par rapport au contenu des messages véhiculés (McKee, 1988 ; Melkote, 2002 ; Snyder, 2002 ; Standing, 2004 ; Kotler et al., 2006).

\section{La sociologie des usages}

Vedel (1994), Vitalis (1994) enrichissent le cadre des théories de la traduction dans leur modèle de «technologie douce». Dans leurs travaux, ils plaident pour une représentation des utilisateurs qui ne serait pas seulement virtuelle (la représentation des usages futurs), mais aussi institutionnelle. Pour les auteurs, les utilisateurs citoyens et institutions publiques - doivent être représentés dans le processus de conception de l'innovation. Paquienséguy (2006, 2007), Proulx (2008, 2015), Badillo et Pélissier (2015), Coutant (2015), pour n'en citer que quelques-uns, mettent en évidence les tensions et les interactions sociotechniques dans la société de l'information et du numérique d'aujourd'hui, en soulignant en même temps le rôle prédominant de l'«utilisateur». Entre autres, il ressort que, malgré les bouleversements techniques permanents de l'époque, l'usage constant des TIC viendrait de " la communication et de sa médiation et non d'une technologie instable " puisque " les machines changent, la médiation reste" (Paquienséguy, 2007) citant Boullier, 2001). Il serait alors " plus fructueux d'étudier dans sa transversalité « le rapport habituel aux objets techniques » (Proulx, 2000) à partir des pratiques communicationnelles des individus » (Paquienséguy, 2007) ; d'étudier "non plus“ ce que les gens font des objets techniques "... mais“ ce que font les gens "... La technologie devient une dimension de l'écologie humaine et sociale parmi d'autres." (Proulx, 2015).

Massit-Folléa (2002) aborde également la sociologie des usages comme «non pas un champ de recherche, mais une préoccupation interdisciplinaire " de la sociologie des technologies, de la communication et des modes de vie. Proulx (2005), quant à lui, identifie cinq courants à considérer dans l'étude des usages : le courant de diffusion sociale de l'innovation ; le courant de la traduction et de l'innovation ; les significations de l'utilisation; action située et cognition distribuée; la sociopolitique des usages. Flichy (2008) observe que " les usages ne peuvent être étudiés qu'à la frontière de plusieurs domaines ": ceux de la sociologie des technologies, la sociologie des médias (réception, usages et récompenses) et l'intégration des TIC dans les sciences de la communication

\section{Instruments et collecte de données}

Dans cette section, les aspects liés aux instruments, à la population étudiée, à l'échantillon, à la méthode et aux techniques de collecte et d'analyse des données et d'interprétation des résultats sont présentés. 

adhésion croissante d'année en année, mais l'année 2020 est l'année qui enregistre beaucoup plus de transactions par LUMICASH. Cela pousse à confirmer que le paiement des taxes par téléphone mobile est une facilité qui connaît une adhésion progressive au Burundi, quoiqu'encore à l'état embryonnaire si l'on considère les milliers de
contribuables qui sont immatriculés (plus de 8000 nouveaux contribuables qui Burundi, quoiqu'encore à l'état embryonnaire si l'on considère les milliers de
contribuables qui sont immatriculés (plus de 8000 nouveaux contribuables qui reçoivent leur numéro d'identification fiscale) au Burundi chaque année.

Les contribuables concernés par les mesures de conformité sont notre population cible. Ils remplissent leurs obligations fiscales en déclarant et en payant les impôts dus. Notre échantillon est cependant constitué de contribuables en cours de paiement (la déclaration n'est pas prise en compte). Le mode d'échantillonnage est aléatoire.

\section{Méthode et approche}

La méthodologie de collecte et d'analyse des données est basée sur une recherche documentaire et sur un échantillon aléatoire de 61 contribuables rencontrés au hasard sur les queues auxquels un questionnaire a été administré au même moment sur trois sites en ville de Bujumbura par des étudiants stagiaires au sein de l'office Burundais des Recettes, Direction en charge de la communication et des Services aux contribuables. Ils s'adressaient à chaque personne en file d'attente afin de connaître la perception des facilités par l'utilisation du téléphone pour le paiement des taxes.

Une question leur a été posée et fait objet de synthèse du questionnaire en annexe : "pourquoi venez-vous faire la queue pour le paiement de la taxe alors qu'une option donnée du paiement par téléphone est accordée par l'administration fiscale ?»

L'analyse du contenu porte sur des données fournies par la plateforme entre l'administration fiscale burundaise et un opérateur de téléphonie mobile opérant au Burundi dénommé LUMITEL, le seul qui permet le paiement des taxes avec son système de transaction appelé LUMICASH. Nous avons d'abord procédé à la lecture des rapports annuels (2017-2020) et des rapports mensuels concernant l'an 2020. L'analyse des données a été effectuée selon une grille d'analyse tridimensionnelle : types de taxe, le nombre de transactions et la comparaison en termes de pourcentage des paiements par téléphone versus par banque. Cette analyse du contenu nous a permis de savoir l'ampleur de l'utilisation du téléphone mobile comme outil de paiement des taxes et sa place comme outil stratégique pour l'administration fiscale.

\section{Résultats}

Les données suivantes permettent de comprendre l'ampleur d'utilisation du téléphone mobile comme moyen de paiement des impôts et taxes.

Graphique 1: Fréquence des paiements des impôts et taxes par téléphone mobile de 2017 à 2020

Avec ce graphique, on voit que le paiement des taxes par téléphone a connu une

Les données suivantes permettent de savoir l'ampleur des montants qui sont collectés au moyen du téléphone, afin de déterminer le profil des contribuables qui préfèrent ce moyen. Soit ce sont de gros montants payés par peu de contribuables et là on déduira qu'il s'agit des grandes entreprises ; soit ce sont de petits montants payés par beaucoup de contribuables et on déduira qu'il s'agit de micro-contribuables. 


\begin{tabular}{|l|l|}
\hline Types de taxes & Nombre de transactions \\
\hline Impôts/Banques & 280224 \\
\hline Impôts/Lumicash & 22898 \\
\hline TOTAL & 303122 \\
\hline
\end{tabular}
payés au moyen du téléphone mobile précédent.

Tableau $1:$ Regroupement selon les types de taxes

\begin{tabular}{|l|l|}
\hline Types d'impôt & Nombre de transactions \\
\hline Actes Administratifs & 72410 \\
\hline Taxes et amendes & 15043 \\
\hline Recettes fiscales & 513 \\
\hline
\end{tabular}

Graphique 2 : Montants encaissés par le Trésor public à travers des impôts et taxes

Avec le tableau et le graphique, on voit que l'administration fiscale a collecté environ 700 millions (BIF) de revenus payés par téléphone en 2020. Ce ne sont pas de sommes importantes en considérant les recettes totales collectées d'année en année (évaluées à plus de 1000 milliards de Fbu). Ces résultats poussent à confirmer que ce ne sont que de micro contribuables (au très bas chiffre d'affaires) qui utilisent plutôt le téléphone pour payer leurs taxes. Le tableau suivant illustre le types de taxes payées par téléphone.

4 En regroupant les taxes et impôts payés au moyen du téléphone mobile, il est question de comprendre le type de taxes et impôts payés et par-là, déterminer davantage le profil des contribuables en complément des informations données par le graphique

5 Ce tableau permet de voir quels types de transactions opérées via le téléphone car cette donnée renseigne sur les catégories des contribuables. Les actes administratifs sont recherchés plus par des étudiants pour des formalités administratives pour recevoir certains documents administratifs, des frais de justice et des autorisations administratives diverses. Du coup, on comprend que ce sont pas des contribuables nécessairement immatriculés ${ }^{12}$ qui utilisent plus le téléphone pour payer les impôts et taxes, mais toute personne en besoin des actes administratifs.

\section{Graphique 3 : Les types d'impôts et taxes payés au moyen du téléphone mobile}

On voit que les actes administratifs sont souvent payés par téléphone plus que les autres types d'impôts, suivent les taxes et pénalités, enfin, les impôts à régime déclaratif (1\%). En effet, cela signifie que le contribuable sous régime déclaratif ne peut pas éviter la queue puisqu'il n'y a pas de télédéclaration. Pour lui, le téléphone n'est pas un outil de facilitation, ce qui explique le faible taux de transactions par téléphone.

\section{Tableau 2 : Regroupement des transactions par banque versus par téléphone en 2020}

Communication, technologies et développement, 10 | 2021 
Ce tableau permet de comparer entre la banque et le téléphone quelle voie la plus préférée pour payer les impôts par les contribuables immatriculées par l'Administration fiscale.

Graphique 4 : Comparaison selon les voies de paiement, banque versus téléphone mobile exprimé leurs appréciations sur la facilitation offerte par le paiement des taxes et impôts au moyen du téléphone mobile.

Tableau 3 : Appréciation positive de la facilité offerte par le paiement des taxes à distance

\begin{tabular}{|l|l|l|}
\hline Facilitation des TIC dans le paiement des taxes & Fréquence & Pourcentage \\
\hline Le paiement des taxes par voie bancaire suffit & 11 & 18.1 \\
\hline Le paiement des taxes à distance est une voie assez exploitée & 13 & 21.3 \\
\hline Le paiement des taxes par téléphone est bien connu & 37 & 60.6 \\
\hline Total & 61 & 100.0 \\
\hline
\end{tabular}

41 Le tableau 3 montre que les participants connaissent dans leur majorité l'usage du téléphone mobile pour payer les taxes tout en affichant un désaccord sur l'exploitation effective des TIC en vue de la promotion du paiement à distance.

\section{Discussion des résultats}

42 Les tableaux et graphiques ci-haut insérés permettent de comprendre que le téléphone comme outil de paiement est d'abord connu par le public en général en considérant le profil des utilisateurs : ce sont des micro contribuables qui sont très nombreux dans le pays, frôlant presque le secteur informel; ensuite les graphiques montrent que ce moyen n'est pas pour autant préféré. Pourquoi ? La discussion des résultats le clarifie.

Nous observons les situations suivantes :

44 - Le téléphone est plus utilisé pour payer les frais d'actes administratifs ( $94 \%$ de toutes les transactions par téléphone sont constituées par des paiements des frais administratifs seulement) ; à ce stade, il faut reconnaitre le grand nombre de paiements de petits montants (pour les certificats, les frais divers,...)

45 - D'un côté, les données sur les transactions des coûts d'actes administratifs ne permettent pas d'identifier les vrais contribuables. De l'autre, les recettes non fiscales et les pénalités sont principalement faites pour les fautes de circulation. Les deux situations ne renseignent pas nécessairement sur la part des vrais contribuables ;

46 - Les contribuables inscrits paient rarement par téléphone (8\% de la taxe fiscale collectée par téléphone), mais utilisent davantage la banque (92\% par banque) 
47 - Les contribuables inscrits qui paient par téléphone sont très peu nombreux (6\% de la taxe fiscale perçue par téléphone) par rapport à l'ensemble des transactions effectuées par téléphone.

Sur la base de ces constats ci-dessus, nous avons cherché à comprendre la raison qui pousse les contribuables à préférer le déplacement vers les guichets des banques pour payer les impôts et taxes et ensuite se présenter aux guichets de réception de l'administration fiscale alors qu'ils pourraient faire la même procédure par téléphone.

Sur 61 personnes auxquelles la question suivante a été adressée : "pourquoi faites-vous la queue alors que le téléphone pourrait vous faciliter la tâche ?" " $92 \%$ savent que payer les impôts par téléphone est fonctionnel au Burundi, mais $3 \%$ l'ont déjà utilisé. Deux raisons de cette méfiance à l'égard de l'utilisation du téléphone sont enregistrées :

«Pas besoin de payer par téléphone si nous devons encore récupérer le reçu comme preuve de paiement aux guichets de l'OBR » : (99\%)

«Le paiement à distance n'est pas fiable » : (1\%)

52 De ce qui précède, nous concluons ce qui suit :

53 1. Les informations sur le paiement des taxes et droits sont bien connues des contribuables ;

54 2. La communication entre l'administration fiscale et les contribuables sur la nouvelle technologie n'est pas complète tant que le retour d'information de l'administration fiscale est quasiment inexistant. En effet, selon McLean, le feedback est le feedback de l'expéditeur dans un jeu sans fin. Le diagramme ci-dessous illustre ce qui doit être fait :

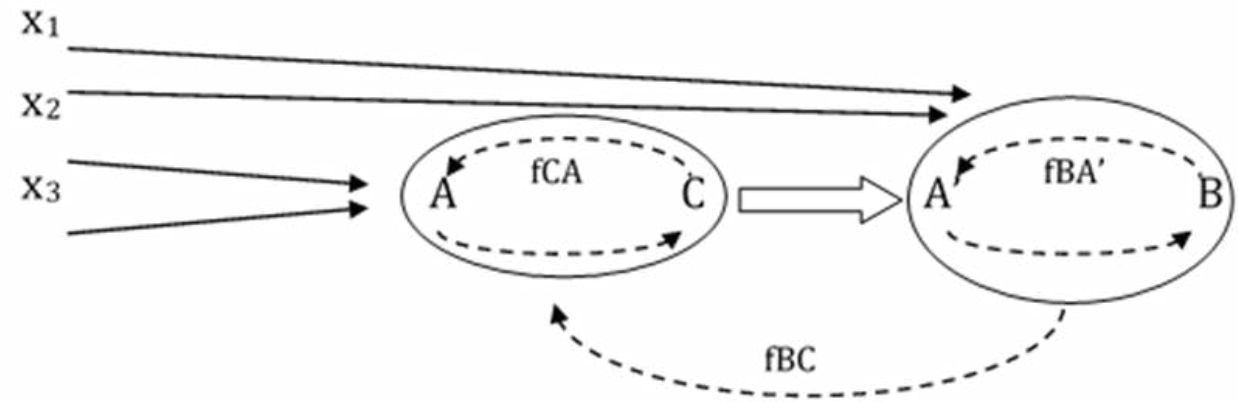

55 Les $\mathrm{X}$ sont les actions de sensibilisation émises lors des campagnes (premier étage " réception d'information ", " mise à l'agenda »). L'administration fiscale a produit une démonstration vidéo visuelle de la méthode de paiement des impôts et des taxes par téléphone et elle est attrayante et convaincante en soi.

Le $\mathrm{fCA}$, le fBA et le $\mathrm{fBC}$ sont les processus d'influence et de construction d'opinions relatives aux nouvelles technologies (deuxième "étape ": appropriation de l'information). $92 \%$ des contribuables ont leur avis, ils estiment qu'il est inutile de payer des impôts par téléphone s'ils doivent revenir faire la queue pour recevoir des reçus ; 3 \% n'y croient pas du tout (peu fiables). En effet, selon la théorie de l'usage, l'appropriation du comportement souhaité ou son rejet peut avoir pour origine la perception de l'outil intermédiaire entre le contribuable et l'État dans l'accomplissement de ses obligations fiscales, sans parler de la menace qu'il peut ressentir venir du nouveau dispositif qui lui est imposé.

57 L'Administration fiscale du Burundi mène des campagnes de sensibilisation (X). Ils touchent directement les contribuables (B), mais aussi leurs associations (C). 
Cependant, mis à part cette vidéo diffusée via l'une des télévisions locales et sur les écrans du service d'accueil, cet avis des contribuables n'est pas encore pris en charge et traité par l'administration fiscale à travers des forums avec des représentants des contribuables pour d'autres aspects tels que la sensibilisation aux nouvelles lois. En effet, les contribuables construisent leur opinion sur la base de ces deux courants d'influence qui se combinent et où interviennent deux niveaux (feedbackBA - feedback CA) des « influenceurs », des leaders d'opinion (A).

L'idée défendue ici est qu'il ne suffit pas de combler le «fossé numérique » dans les TIC. Les appréciations des contribuables comptent beaucoup. Vedel (1994), Vitalis (1994) enrichissent le cadre des théories de la traduction dans leur modèle de «soft technology ». Dans leurs travaux, ils plaident pour une représentation des utilisateurs qui ne serait pas seulement virtuelle (la représentation des usages futurs), mais aussi institutionnelle. Pour les auteurs, les utilisateurs - citoyens et institutions publiques doivent être représentés dans le processus de conception de l'innovation.

Il est donc clair, après avoir observé les files d'attente persistantes, que la facilitation par téléphone n'est pas complète tant qu'il n'y a pas de fonctionnalité permettant de numériser les reçus comme preuve de paiement. Les contribuables devraient les recevoir sur leur téléphone à des fins administratives. Cependant, comme le soulignent les auteurs, le rôle de l'utilisateur dans le processus de numérisation est primordial: pour n'en citer que quelques-uns, mettre en évidence les tensions et les interactions sociotechniques dans la société de l'information et du numérique d'aujourd'hui, tout en soulignant le rôle prédominant des « utilisateurs » (Pasquienséguy $(2006,2007)$, Proulx (2008, 2015), Badillo et Pélissier (2015), Coutant (2015). Entre autres, il ressort que, malgré les bouleversements techniques permanents de l'époque, la constante l'utilisation des TIC proviendrait de « la communication et de sa médiation et non d'une technologie instable " puisque "les machines changent, la médiation demeure " (Paquienséguy, 2007) citant Boullier, 2001). Il serait alors plus fructueux d'étudier dans sa transversalité « le rapport habituel aux objets techniques "(Proulx, 2000) à partir des pratiques communicationnelles des individus " (Paquienséguy, 2007); d'étudier "non plus" ce que les gens font des objets techniques "... mais" ce que les gens font "..." (Proulx, 2015).

Notre hypothèse citée ci-dessus : « les contribuables burundais ne sont pas pour autant facilités avec l'introduction du téléphone mobile comme moyen de paiement des impôts et taxes. » est confirmée compte tenu de l'analyse des données sur la petite taille des contribuables inscrits qui utilisent le téléphone pour payer les impôts. A l'inverse, nous avons observé un très grand nombre de personnes anonymes qui paient les frais administratifs par téléphone. En effet, l'administration fiscale n'a pas encore réalisé complètement la mise en œuvre du téléphone comme outil de renforcement de la conformité fiscale en offrant des services en ligne, aussi longtemps qu'il n'y aura pas eu digitalisation de la quittance comme preuve de paiements et de la télédéclaration des impôts.

61 Notre étude conclut que les contribuables burundais n'ont pas adopté la facilitation par le téléphone mobile, qui est plutôt perçu comme un outil qui accélère plus la collecte des recettes que la facilitation des contribuables. 


\section{BIBLIOGRAPHIE}

Albarello, L. (1999). Apprendre à chercher. De Boeck.

Amah.,E. C. M. (2015). L'utilisation du téléphone mobile chez les pêcheurs et les marchands des produits de la pêche artisanale maritime au port de Lomé. dans A. Kiyindou, K. Anaté, A. CapoChichi, Quand l'Afrique réinvente la téléphonie mobile, L'Harmattan, p. 219-233.

Amy, Q. et al. (2020). Mobile phone use is associated with higher smallholder agricultural productivity in Tanzania. East Africa, Plos one.

Bardin, L. (1989). L'analyse de contenu dans l'étude des représentations sociales. Sociologies. Banque Mondiale. (2012). Information and Communications for Development: Maximizing Mobile, The World Bank Group, Washington DC.

https://openknowledge.worldbank.org/handle/10986/11958

Banque Mondiale. (2016). Les dividendes du numérique. Rapport sur le développement dans le monde, The World Bank Group, Washington DC.

https://www.worldbank.org/en/publication/wdr2016

Banque Mondiale. (2018a). Rapport sur la pauvreté et la prospérité partagée. Compléter le puzzle de la pauvreté, The World Bank Group, Washington DC.

https://openknowledge.worldbank.org/handle/10986/30418 ?show =full\&locale-attribute $=$ fr Castells, M. (1997). The Information Age : Economy, Society and Culture vol. II : The Power of Identity. Malden MA. Oxford (UK), Blackwell Publishers.

Castells, M. (2000). The Information Age : Economy, Society and Culture vol. I (second edition) : The Rise of the Network Society. Cambridge MA. Oxford UK, Blackwell.

Cheneau-Loquay. A. (2010a). L'Afrique au seuil de la révolution des télécommunications. Les grandes tendances de la diffusion des TIC. Afrique contemporaine, $\mathrm{n}^{\circ} 234, \mathrm{pp}$. 93-112

Dereze. G. (2009). Méthodes empiriques de recherche en communication, De Boeck, Bruxelles.

Everett M. R \& Adhikarya. R. (1979). Diffusion of Innovations : An Up-To-Date Review and Commentary. Annals of the International Communication Association, Volume (3), pages 67-81

Gina. P. et al. (2015). Mobile Phones and Education in Sub-Saharan Africa : From Youth Practice to Public Policy, Journal of International Development. Pages 22-39

Grawitz. M. (1991). Méthodes des sciences sociales, Dalloz.

Jeffrey. J. \& Mila V. (2007). Mobile phones in Africa : how much do we really know ?, Springer Open Choice. Pages 117-126

Kiyindou. A. La lutte contre la pauvreté et/ou pratique des TIC, Communication \& langages 2010/1 $\left(\mathrm{N}^{\circ} 163\right)$. Pages 121-133

Loquay. A. La téléphonie mobile dans les villes africaines. Une adaptation réussie au contexte local. Espace géographique, 2012/1 Tome 41 | pages 82 à 93

Velmuradova. M. (2015). Communication pour le développement et l'intégration sociale de nouveaux dispositifs : le rôle de la valeur perçue d'usage. Étude de cas dans l'appui à des Petites et moyennes entreprises au Turkménistan. 
https://hal.archives-ouvertes.fr/tel-01582290/

Wilbur. S. (2011). Beginnings of the Communication. Communication Research Trends, Volume 6

Wilkins, K. G., \& Mody, B. (2001). Reshaping development communication : Developing

communication and communicating development. Communication Theory, 11(4), 385-396.

SERVAES. J. (2007). Communication for development and social change, Sage.

Simplice. A. (2015). The Impact of Mobile Phone Penetration on African Inequality, International Journal of Social Economics.

\section{NOTES}

1. Selon le Rapport de GSM Association (2017), l'Afrique subsaharienne comptait à elle seule 420 millions d'abonnés uniques en 2016 , soit un taux de pénétration de $43 \%$. Selon les projections, ce taux devrait atteindre les $50 \%$ en 2020, avec 535 millions d'abonnés en Afrique subsaharienne et 725 millions pour tout le continent.

2. Nous avons plutôt privilégié une vision large du sujet (à l'échelle africaine) parce que, d'une façon générale, en matière de développement (ou de sous-développement), les pays africains ne se distinguent pas fondamentalement les uns des autres. Les problèmes sont presque identiques partout, à quelques rares exceptions près (l'Afrique du Sud notamment et le Maghreb dans une moindre mesure). Surtout quand il s'agit du taux de pénétration des TIC dans chaque pays en général et l'usage du téléphone mobile comme outil de toute sorte de transaction en particulier. Pour autant, les données dans la présente étude portent sur le contexte burundais qui résume bien le contexte de la plupart des pays africains, pour les raisons évoquées.

3. Elisabeth Peyroux et Olivier Ninot, La Révolution numérique en Afrique, article publié dans « Parole d'Expert », 1 juillet 2019

4. Annie Chéneau-Loquay, La téléphonie mobile dans les villes africaines. Une adaptation réussie au contexte local, Revue « Espace géographique, 2012/1 Tome 41 | pages 82 à 93

5. Jean-Philippe Berrou et Kevin Mellet, Une révolution mobile en Afrique subsaharienne?

6. À titre d'exemple, la plateforme de paiement numérique M-Pesa, proposée par Safaricom au Kenya depuis 2007, a connu une croissance rapide, illustrant la mutualisation des infrastructures et l'interopérabilité entre les grands opérateurs.

7. La transformation numérique en Afrique subsaharienne ...www.imf.org > AFR > 2020 > April

8. Rapport trimestriel de performance de l'Office Burundais des Recettes, avril-juin 2019.

9. En 2019, l'Office Burundais des Recettes a signé une convention de partenariat avec la compagnie de téléphonie mobile LUMITEL pour le paiement des impôts et taxes à travers son réseau téléphonique

10. Thèse publié par Maya VELMURADOVA: Communication pour le développement et l'intégration sociale de nouveaux dispositifs : le rôle de la valeur perçue d'usage. Étude de cas dans l'appui à des Petites et moyennes entreprises au Turkménistan.

11. Idem

12. Au Burundi, pour être appelé contribuable, il faut se faire immatriculer et obtenir un identifiant fiscal ainsi qu'un compte courant fiscal. Seul le paiement des frais administratifs échappe à cette règle. 


\section{RÉSUMÉS}

Les Technologies de l'Information et de Communication (TIC) ont un impact significatif sur le comportement des contribuables dans les pays aux moyens économiques limités, dont l'Afrique subsaharienne. Même si l'Afrique apparaît encore dans le bas des classements mondiaux en matière de développement des technologies de l'information et de la communication (TIC) et de l'exploitation de leur potentiel, la diffusion de la téléphonie mobile sur le continent, qui date de la fin des années 1990, s'est cependant fortement accélérée au cours des dernières années. ${ }^{1}$

Le paiement des impôts et taxes au moyen du téléphone mobile, sujet qui occupe cet article, rentre dans cette cadence d'opportunités offertes par l'outil, mais reste limité à un petit nombre de contribuables (résidant en ville) tandis qu'une grande majorité de petits et moyens contribuables restent dispersés dans les zones semi-urbaines des provinces. Ces derniers vivent dans des paradis fiscaux en raison du manque de moyens des autorités fiscales pour les localiser, les enregistrer et les taxer. C'est là que les TIC trouvent leur place. ${ }^{2}$

En conséquence, la communication pour la promotion du civisme fiscal n'est pas suffisante sans le support de solutions informatiques mises à disposition des contribuables d'une part et sans le soutien de campagnes de sensibilisation (communication de masse avec l'aide des médias) d'autre part pour inciter les contribuables à les utiliser et les adopter.

Le présent article porte sur l'exploitation de l'opportunité du téléphone mobile comme moyen de booster le civisme fiscal grâce à l'expérience gratifiante de facilitation et de rapidité des transactions entre le contribuable et le fisc.

Information and Communication Technologies (ICTs) have a significant impact on the behavior of taxpayers in countries with limited economic means, including sub-Saharan Africa. Even though Africa still appears at the bottom of the world rankings for the development of information and communication technologies (ICT) and the exploitation of their potential, the spread of mobile telephony on the continent, which date of the late 1990s, however, has accelerated sharply in recent years.

The payment of taxes and duties by means of the mobile telephone, subject which occupies this article, falls within this rate of opportunities offered by the tool, but remains limited to a small number of taxpayers (residing in town) while a large number of taxpayers the majority of small and medium taxpayers remain dispersed in the semi-urban areas of the provinces. The latter live in tax havens due to the lack of resources of the tax authorities to locate, register and tax them. This is where ICTs find their place.

Consequently, communication for the promotion of tax compliance is not sufficient without the support of IT solutions made available to taxpayers on the one hand and without the support of awareness campaigns (mass communication with the help of the media) on the other hand, to encourage taxpayers to use and adopt them.

This article focuses on harnessing the opportunity of the mobile phone as a means of boosting tax compliance through the rewarding experience of facilitating and speeding transactions between taxpayer and Revenue authority.

\section{INDEX}

Mots-clés : Téléphone, conformité, facilitation

Keywords : Telephone, conformity, facilitation 
AUTEUR

FIACRE MUHIMPUNDU

École Doctorale de l'Université du Burundi 